\title{
ARTICLE
}

\section{Xenon-mediated neuroprotection in response to sustained, low-level excitotoxic stress}

\author{
J Lavaur ${ }^{1}$, M Lemaire ${ }^{2}$, J Pype $^{2}$, D Le Nogue ${ }^{1}$, EC Hirsch ${ }^{1}$ and PP Michel ${ }^{1}$
}

Noble gases such as xenon and argon have been reported to provide neuroprotection against acute brain ischemic/anoxic injuries. Herein, we wished to evaluate the protective potential of these two gases under conditions relevant to the pathogenesis of chronic neurodegenerative disorders. For that, we established cultures of neurons typically affected in Alzheimer's disease (AD) pathology, that is, cortical neurons and basal forebrain cholinergic neurons and exposed them to L-trans-pyrrolidine-2,4-dicarboxylic acid (PDC) to generate sustained, low-level excitotoxic stress. Over a period of 4 days, PDC caused a progressive loss of cortical neurons which was prevented substantially when xenon replaced nitrogen in the cell culture atmosphere. Unlike xenon, argon remained inactive. Xenon acted downstream of the inhibitory and stimulatory effects elicited by PDC on glutamate uptake and efflux, respectively. Neuroprotection by xenon was mimicked by two noncompetitive antagonists of NMDA glutamate receptors, memantine and ketamine. Each of them potentiated xenon-mediated neuroprotection when used at concentrations providing suboptimal rescue to cortical neurons but most surprisingly, no rescue at all. The survival-promoting effects of xenon persisted when NMDA was used instead of PDC to trigger neuronal death, indicating that NMDA receptor antagonism was probably accountable for xenon's effects. An excess of glycine failed to reverse xenon neuroprotection, thus excluding a competitive interaction of xenon with the glycine-binding site of NMDA receptors. Noticeably, antioxidants such as Trolox and N-acetylcysteine reduced PDC-induced neuronal death but xenon itself lacked free radical-scavenging activity. Cholinergic neurons were also rescued efficaciously by xenon in basal forebrain cultures. Unexpectedly, however, xenon stimulated cholinergic traits and promoted the morphological differentiation of cholinergic neurons in these cultures. Memantine reproduced some of these neurotrophic effects, albeit with less efficacy than xenon. In conclusion, we demonstrate for the first time that xenon may have a therapeutic potential in AD.

Cell Death Discovery (2016) 2, 16018; doi:10.1038/cddiscovery.2016.18; published online 16 May 2016

\section{INTRODUCTION}

Noble gases such as xenon and argon have little propensity to participate in chemical reactions because of a filled valence shell. However, both xenon and argon possess interesting biological properties. Xenon is an approved anesthetic drug ${ }^{1,2}$ with organoprotective properties when administered alone ${ }^{3,4}$ or in combination with hypothermia. ${ }^{5,6}$ Xenon has been described as neuroprotectant in preclinical models of focal and global brain ischemia, ${ }^{7-11}$ spinal cord ischemia ${ }^{12}$ and traumatic brain injury. ${ }^{13,14}$ Some of the organoprotective and neuroprotective properties of xenon are also shared by argon. ${ }^{14-16}$ For example, argon can provide neuroprotection in acute brain slices subjected to oxygen and glucose deprivation, ${ }^{16}$ in rats subjected to intrastriatal injection of N-methyl-D-aspartate (NMDA) ${ }^{16}$ or to transient occlusion of the middle cerebral artery. ${ }^{17}$ Most recently, argon was also reported to reduce apoptosis of retinal ganglion cells after ischemia/reperfusion injury of the rat's eye. ${ }^{18}$

Activation of ATP-sensitive potassium channels or of two-pore potassium channels may explain some of the neuroprotective effects of xenon. ${ }^{19,20}$ Yet, it appears that xenon primarily acts by preventing NMDA receptor overexcitation under excitotoxic stress conditions. $^{7,14,21}$ More specifically, xenon was reported to exert competitive inhibition at the glycine site of the NMDA receptor. ${ }^{22}$ Argon is protective too in experimental situations where neurodegenerative changes result from NMDA receptor overexcitation. However, argon distinguishes itself from xenon by not directly interfering with NMDA receptors and the putative targets of argon are still yet unknown. ${ }^{15,23}$

Excitotoxic stress mediated through NMDA receptors is most frequently associated to acute central nervous system insults such as ischemia and traumatic brain injury but chronic low-level overexcitation of these receptors by glutamate is also suspected to be one of the factors contributing to neuronal death in a number of chronic neurodegenerative conditions, including amyotrophic lateral sclerosis, Parkinson's disease and Alzheimer's disease (AD). ${ }^{24,25}$ The possible role of excitotoxic stress in $A D$ is also suggested by reports showing that the two NMDA receptor antagonists, memantine and its close derivative nitromemantine provide some protection against $A D$ progression in animal models of the disease. ${ }^{26}$ Memantine has also a small clinically detectable effect on cognitive dysfunction in AD patients. ${ }^{27}$

In the present study, we wished to assess the neuroprotective potential of xenon and argon in conditions that mimic chronic low-level excitotoxic stress as it may occur in AD. To this aim, we established cultures of neuronal populations most vulnerable in $A D$, that is, cortical neurons $s^{28-30}$ and basal forebrain cholinergic neurons. ${ }^{29,31,32}$ These cultures were submitted to mild excitotoxic stress by continued exposure to L-trans-pyrrolidine-2,4-dicarboxylic acid (PDC), a synthetic analog of L-glutamate that exerts

${ }^{1}$ Institut National de la Santé et de la Recherche Médicale, U 1127, CNRS, Unité Mixte de Recherche (UMR) 7225, Sorbonne Universités, UPMC Univ Paris 06, UMR S 1127, Institut du Cerveau et de la Moelle épinière, ICM, Paris, France and ${ }^{2}$ Air Liquide Santé International Medical R\&D Paris, Saclay Research Center, Jouy-en Josas, France. 
inhibitory and stimulatory effects on glutamate uptake and efflux, respectively. ${ }^{33-35}$

We demonstrate for the first time that xenon can provide partial but sustained protection to cortical neurons undergoing neurodegeneration through mild excitotoxic stress. These protective effects were mimicked and amplified by two noncompetitive NMDA glutamate receptor antagonists, memantine and ketamine. Xenon also provided robust protection to PDC-treated cholinergic neurons in septal cultures but quite unexpectedly, it also exerted potent neurotrophic effects on these neurons.

\section{RESULTS}

Xenon, but not argon, protects cortical neurons from death induced by chronic exposure to PDC

Cortical cultures that had initially matured in vitro for 12 days were exposed to PDC, the synthetic analog of glutamate, to induce neurodegeneration. Cortical neurons identified by their content in microtubule-associated protein-2 (MAP-2) were affected when PDC was applied at concentrations equal or greater than $30 \mu \mathrm{M}$ for four consecutive days (Figure 1a). In a time course experiment carried out with $30 \mu \mathrm{M}$ PDC, we observed that neurodegeneration affected about $45 \%$ of cortical neurons after 1 day and more than $80 \%$ after 4 days of treatment (Figure $1 \mathrm{~b}$ ), indicating that neuronal demise developed progressively as a function of time in this experimental setting.

PDC-treated cortical neurons were largely protected from neurodegeneration when $75 \%$ nitrogen contained in the standard gas atmosphere was replaced by $75 \%$ xenon. After 1 and 4 days of treatment with $30 \mu \mathrm{M}$ PDC, the survival rate of cortical neurons in cultures chronically exposed to xenon was $89 \%$ (versus $55 \%$ in $\mathrm{N}_{2}$ ) and $62 \%$ (versus $20 \%$ in $\mathrm{N}_{2}$ ), respectively (Figures $1 \mathrm{~b}$ and c). After 4 days of incubation with $100 \mu \mathrm{M}$ PDC, that is, treatment conditions where neuronal survival is below $5 \%$, xenon remained neuroprotective even if its efficacy was reduced (Figure 1a). The replacement of nitrogen by argon failed to provide protection to cortical neurons undergoing chronic neurodegeneration regardless of the concentration of PDC used (Figure 1a). In the following experiments, we aimed to further characterize the neuroprotective effects of xenon.

The effect of xenon is mimicked by pharmacological blockade of NMDA receptors

To investigate the mechanisms underlying neuroprotection by xenon, we compared the effects of the noble gas to those of memantine, a noncompetitive antagonist of NMDA receptors. At an optimal concentration of $10 \mu \mathrm{M}$, memantine protected about $90 \%$ of cortical neurons exposed for 4 days to $30 \mu \mathrm{M}$ PDC under a standard nitrogen atmosphere (Figure 2a), pointing to a key role of NMDA receptors in PDC-induced neurodegeneration. Xenon rescued $66 \%$ of cortical neurons in the same experimental setting. Glycine $(1 \mathrm{mM})$, a co-agonist with glutamate at NMDA receptors did not intensify PDC-induced neuronal death under a control atmosphere and it also failed to reverse xenon-mediated neuroprotection (Figure 2a). When NMDA $(100 \mu \mathrm{M})$, the preferential agonist of NMDA receptors was used to trigger cortical cell death instead of PDC, xenon remained partially protective (Figure $2 \mathrm{~b})$. Memantine $(10 \mu \mathrm{M})$ rescued more than $89 \%$ of cortical neurons in the presence of $100 \mu \mathrm{M}$ NMDA. The efficacy of xenon to rescue cortical neurons against NMDA $(100 \mu \mathrm{M})$ or PDC $(30 \mu \mathrm{M})$ was roughly similar (Figure $2 \mathrm{~b}$ ).

Xenon and NMDA receptor antagonists protect cortical neurons in a cooperative manner

Memantine and xenon are known to act as blockers of NMDA receptors. $^{26,36}$ Therefore, we investigated whether the two

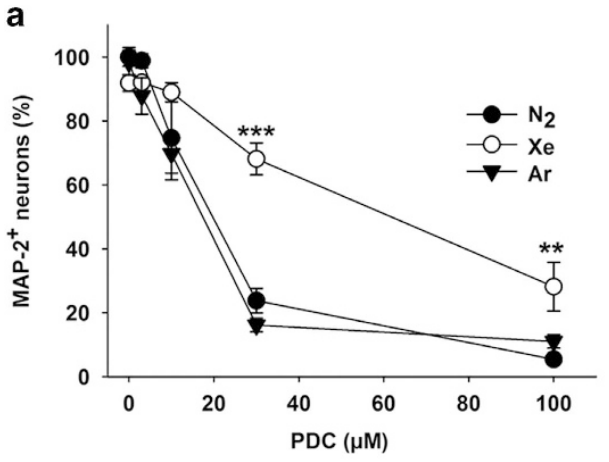

b

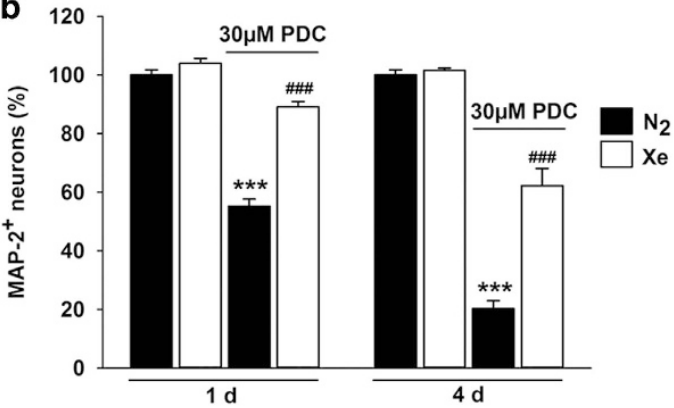

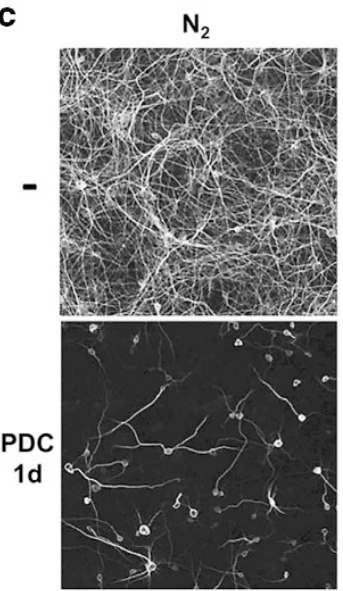

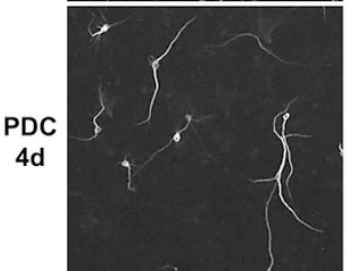

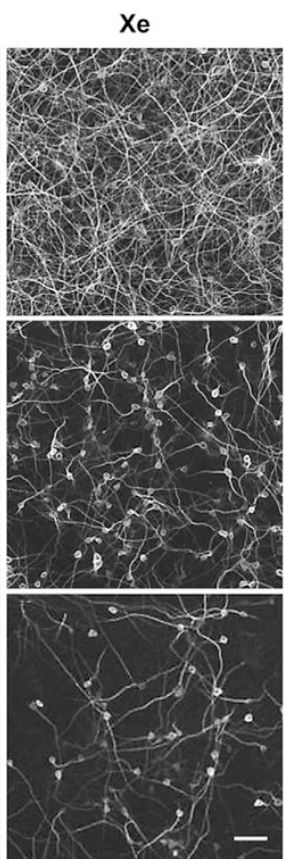

Figure 1. Xenon but not argon is protective against neuronal death induced by PDC in cortical cultures. (a) Survival rate of cortical neurons (MAP-2 ${ }^{+}$cells) in cultures exposed or not to PDC (3-100 $\mu \mathrm{M}$ ) for 4 days under an atmosphere containing $75 \% \mathrm{~N}_{2}, 75 \%$ Xe or $75 \%$ Ar. Error bars indicate mean \pm S.E.M. $(n=9)$. ${ }^{* * P}<0.01,{ }^{* * *} P<0.001$ relative to cultures receiving a same concentration of PDC under $N_{2}$ atmosphere. (b) Survival of cortical neurons exposed to $30 \mu \mathrm{M}$ PDC for 1 or 4 days in an atmosphere containing $75 \% \mathrm{~N}_{2}$ or $75 \%$ Xe. Error bars indicate mean \pm S.E.M. $(n=9)$. ${ }^{* *} P<0.001$ relative to control cultures maintained under $\mathrm{N}_{2}$ atmosphere and ${ }^{\sharp \#} P<0.001$ relative to age-matched PDCtreated cultures under $\mathrm{N}_{2}$ atmosphere. (c) Fluorescence digitized images illustrating the neuroprotective effects provided by a gas atmosphere containing $75 \%$ Xe (versus $75 \% \mathrm{~N}_{2}$ ) in cortical cultures exposed for 1 or 4 days to $30 \mu \mathrm{M}$ of PDC. Scale bar $50 \mu \mathrm{m}$. 


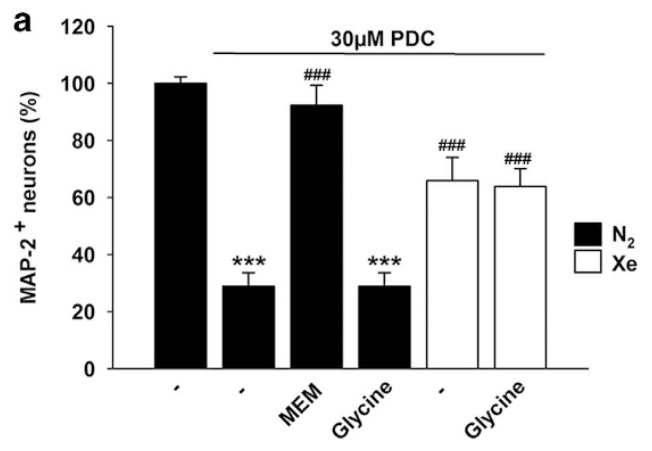

d
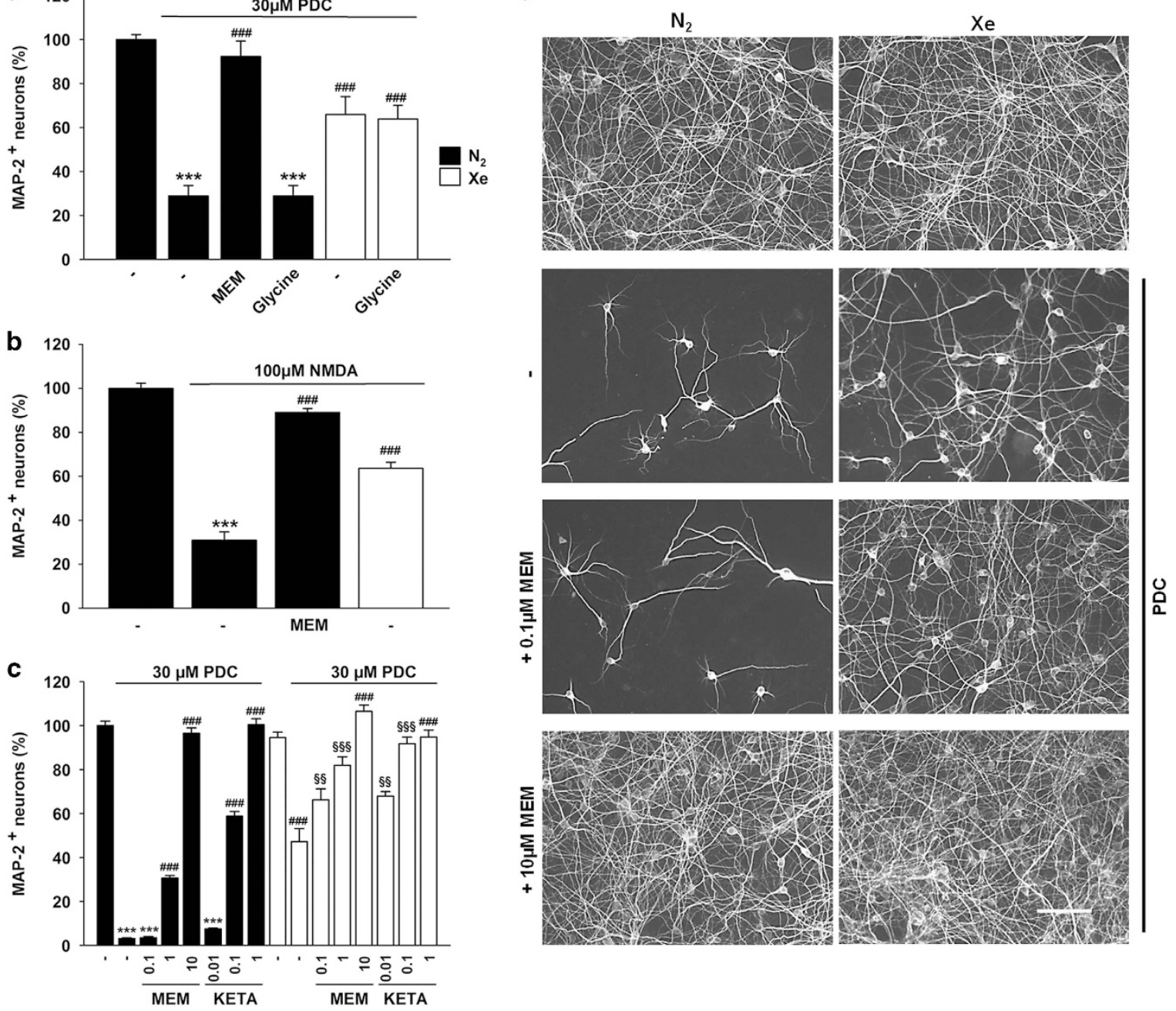

Figure 2. Role of NMDA glutamate receptors in xenon-mediated neuroprotection. (a) Survival rate of cortical neurons exposed to PDC (30 $\mu \mathrm{M}$ ) for 4 days and treated concurrently or not with the NMDA receptor blocker memantine (MEM, $10 \mu \mathrm{M})$ or the amino acid glycine (1 mM) under a gas atmosphere containing $75 \% \mathrm{~N}_{2}$. Some cultures treated with PDC were also placed under a gas atmosphere containing $75 \%$ Xe and treated with or without glycine. Error bars indicate mean \pm S.E.M. $(n=9)$. ${ }^{* * *} P<0.001$ relative to control cultures under $N_{2}$ atmosphere and $\# \# \#<0.001$ relative to PDC-treated cultures under $N_{2}$ atmosphere. (b) Survival of cortical neurons exposed to NMDA (100 $\mu$ M) for 4 days under $75 \% \mathrm{~N}_{2}$ and the impact of a treatment with memantine $(10 \mu \mathrm{M})$ or $75 \%$ Xe. Error bars indicate mean \pm S.E.M. $(n=9)$. ${ }^{* * * P}<0.001$ relative to control cultures maintained under $\mathrm{N}_{2}$ atmosphere and ${ }^{\# \# \# P}<0.001$ relative to PDC-treated cultures under $\mathrm{N}_{2}$ atmosphere. (c) Survival of cortical neurons exposed to PDC $(30 \mu \mathrm{M})$ for 4 days in the presence or not of various concentrations of memantine $(0.1 ; 1 ; 10 \mu \mathrm{M})$ or ketamine $(0.01 ; 0.1 ; 1 \mu \mathrm{M})$ under gas atmospheres containing either $75 \% \mathrm{~N}_{2}$ or $75 \%$ Xe. Error bars indicate mean \pm S.E.M. $(n=9)$. ${ }^{* * * P}<<0.001$ relative to


PDC-treated cultures maintained under $75 \%$ Xe and PDC-treated cultures maintained under $75 \% \mathrm{~N}_{2}$ with a same concentration of memantine. (d) Fluorescence digitized images showing the impact that treatments with xenon alone or xenon+memantine $(0.1 ; 10 \mu \mathrm{M})$ exert on the survival of cortical cultures exposed to PDC. Scale bar $50 \mu \mathrm{m}$.

molecules could cooperate to increase protection of cortical neurons (Figure 2c). Memantine at $0.1 \mu \mathrm{M}$ did not protect cortical neurons, but was able to substantially reinforce the action of xenon. The effect of xenon was also significantly improved with memantine at $1 \mu \mathrm{M}$, a concentration that rescues in itself a substantial fraction of cortical neurons. No improvement of the effect of xenon was observed, however, when memantine was used at $10 \mu \mathrm{M}$, that is, a concentration which already provides optimal neuroprotection per se. Of interest, another NMDA receptor antagonist ketamine also exerted cooperative protective effects with xenon (Figure 2c) when used at concentrations of 0.01 and $0.1 \mu \mathrm{M}$ that exert no and partial protective effects on their own, respectively. No cooperative effects were observed with a concentration of $1 \mu \mathrm{M}$ ketamine that was already optimally protective in itself.
Impact of xenon on the uptake and release of glutamate

We studied whether some of the effects of xenon resulted from direct interference with the mechanism of action of PDC. To do so, we used a non-metabolizable analog of L-glutamate, $\left[{ }^{3} \mathrm{H}\right]-\mathrm{D}$ aspartate, that labels the cytosolic and vesicular pools of endogenous excitatory amino acids. ${ }^{37}$ Our data show that an acute application of PDC $(30 \mu \mathrm{M})$ reduced the uptake of $\left[{ }^{3} \mathrm{H}\right]-\mathrm{D}$ aspartate by more than $70 \%$. The uptake of $\left[{ }^{3} \mathrm{H}\right]-\mathrm{D}$-aspartate was not restored by xenon and it also remained impaired in the presence of memantine $(10 \mu \mathrm{M})$ (Figure 3a). Noticeably, the uptake of $\left[{ }^{3} \mathrm{H}\right]$-D-aspartate was not significantly affected by xenon in control conditions.

Using cortical cultures initially pre-loaded with $\left[{ }^{3} \mathrm{H}\right]$-D-aspartate, we found that PDC $(30 \mu \mathrm{M})$ stimulated the release of the tritiated neurotransmitter, which suggests that neuroprotection by xenon 

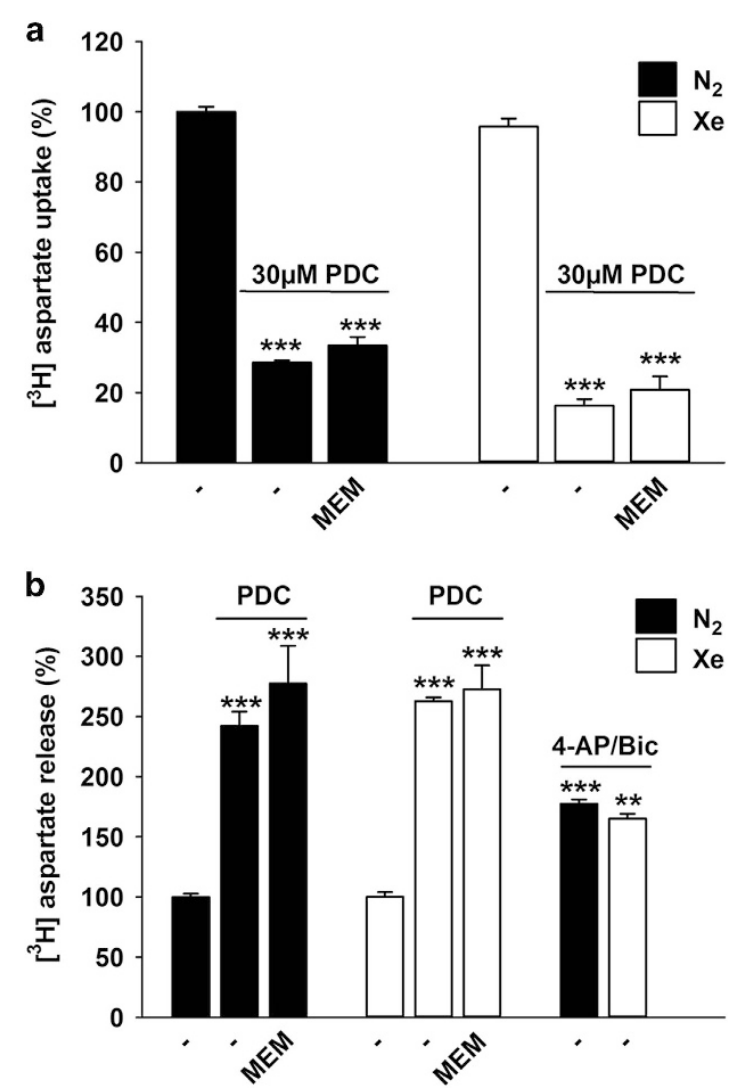

Figure 3. Impact of xenon on the uptake and release of $\left[{ }^{3} \mathrm{H}\right]-\mathrm{D}$ aspartate in cortical cultures exposed to PDC. (a) $\left[{ }^{3} \mathrm{H}\right]$-D-aspartate uptake measured in cortical cultures exposed acutely or not to PDC $(30 \mu \mathrm{M})$ under an atmosphere containing $75 \% \mathrm{~N}_{2}$ or $75 \% \mathrm{Xe}$, in the presence or not of memantine $(10 \mu \mathrm{M})$. Error bars indicate mean \pm S.E.M. $(n=6)$. ${ }^{* * *} P<0.001$ relative to control cultures under $\mathrm{N}_{2}$ atmosphere. (b) $\left[{ }^{3} \mathrm{H}\right]$-D-aspartate released in cortical cultures exposed or not to PDC ( $30 \mu \mathrm{M})$ in an atmosphere containing $75 \% \mathrm{~N}_{2}$ or $75 \% \mathrm{Xe}$, in the presence or not of memantine $(10 \mu \mathrm{M})$. Error bars indicate mean \pm S.E.M. $(n=9)$. ${ }^{* * * P}<0.001$ relative to control cultures under $\mathrm{N}_{2}$ atmosphere. Impact of an atmosphere containing $75 \%$ Xe on $\left[{ }^{3} \mathrm{H}\right]$-D-aspartate release evoked by a depolarizing treatment with 4-aminopyridine (4-AP; $2.5 \mathrm{mM})$ and bicuculline (Bic; $50 \mu \mathrm{M})$. Error bars indicate mean \pm S.E.M. $(n=9) .{ }^{* *} P<0.01$, ${ }^{* * * P}<0.001$ relative to control cultures maintained under $\mathrm{N}_{2}$ atmosphere.

could potentially result from a reduction of PDC-evoked glutamate release. Xenon failed, however, to reduce the efflux of $\left[{ }^{3} \mathrm{H}\right]-\mathrm{D}$ aspartate upon PDC exposure (Figure 3b). Likewise, memantine did not interfere significantly with the release of $\left[{ }^{3} \mathrm{H}\right]$-D-aspartate after PDC treatment.

We also assessed the impact of xenon on $\left[{ }^{3} \mathrm{H}\right]$-D-aspartate release evoked by the $\mathrm{K}^{+}$channel blocker 4-aminopyridine $(2.5 \mathrm{mM})$ and the $\mathrm{GABA}_{\mathrm{A}}$ receptor antagonist bicuculline $(50 \mu \mathrm{M})$, a pharmacological treatment known to physiologically activate neuronal glutamatergic synaptic activity. ${ }^{38,39}$ As expected, the depolarizing treatment enhanced $\left[{ }^{3} \mathrm{H}\right]$-D-aspartate efflux but xenon failed to interfere with this process, which signifies that the noble gas had no significant impact on presynaptic glutamatergic release, in present conditions.

Xenon prevents a death mechanism involving oxidative stress We established that oxidative stress was part of the death process inhibited by xenon. Indeed, the protection provided by $75 \%$ xenon against PDC $(30 \mu \mathrm{M})$ was reproduced partially by Trolox


Figure 4. Xenon prevents a death mechanism that implicates oxidative stress. (a) Survival of cortical neurons in cultures exposed or not to PDC $(30 \mu \mathrm{M})$ for 4 days and treated or not concomitantly with Trolox $(10 \mu \mathrm{M})$ or NAC $(30 \mu \mathrm{M})$ under an atmosphere containing $75 \% \mathrm{~N}_{2}$. Comparison with cultures receiving the same concentration of PDC under an atmosphere containing $75 \%$ Xe. Error bars indicate mean \pm S.E.M. $(n=6) .{ }^{* * *} P<0.001$ relative to control cultures maintained under $75 \% \mathrm{~N}_{2}$ and ${ }^{\#} P<0.05,{ }^{\# \#} P<0.001$ relative to PDC-treated cultures maintained under $75 \% \mathrm{~N}_{2}$. (b) DPPH radical scavenging assay comparing the antioxidant potential of Trolox $(10 \mu \mathrm{M}), \mathrm{NAC}(30 \mu \mathrm{M})$ and memantine $(10 \mu \mathrm{M})$ under $75 \% \mathrm{~N}_{2}$ to that of an atmosphere containing $75 \%$ xenon. Error bars indicate mean \pm S.E.M. $(n=12) .{ }^{* *} P<0.001$ relative to controls maintained under $75 \% \mathrm{~N}_{2}$.

$(10 \mu \mathrm{M})$, a water soluble analog of vitamin $\mathrm{E}$ that operates as a scavenger of hydroxyl radicals ${ }^{40}$ and to a much larger extent by $\mathrm{N}$-acetylcysteine (NAC) $(30 \mu \mathrm{M})$, a precursor of the intracellular antioxidant tripeptide glutathione (Figure $4 a){ }^{41}$ To determine whether xenon had in itself the potential to operate as a free radical scavenger, we tested its activity using the 2-diphenyl-1picrylhydrazyl (DPPH) cell-free assay. ${ }^{42}$ As expected, both Trolox $(10 \mu \mathrm{M})$ and NAC $(30 \mu \mathrm{M})$ reduced DPPH spontaneous oxidation. Their antioxidant capacity also matched their neuroprotective activity (Figures $4 a$ and b). At variance, xenon did not demonstrate free radical scavenging activity (Figure $4 b$ ). Similarly, memantine $(10 \mu \mathrm{M})$ was totally ineffective in this assay.

Xenon exerts both trophic and survival-promoting effects towards septal cholinergic neurons

We also characterized the effects of xenon on septal cholinergic neurons, that is, a subpopulation of forebrain neurons particularly vulnerable to degeneration in the context of AD. ${ }^{31,32}$ Unexpectedly, cholinergic neurons identified by immunofluorescence detection of choline acetyltransferase (ChAT) exhibited much larger cell bodies when exposed transiently to $75 \%$ xenon between 12 and 16 days in vitro (DIV) (Figure 5a, left panel; 


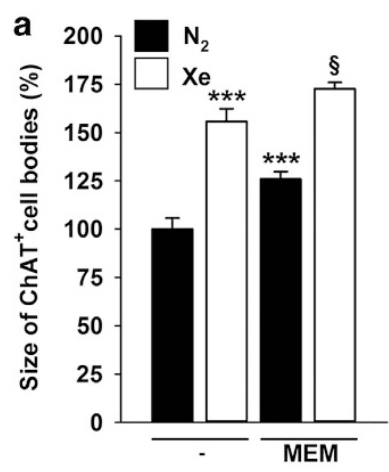

b



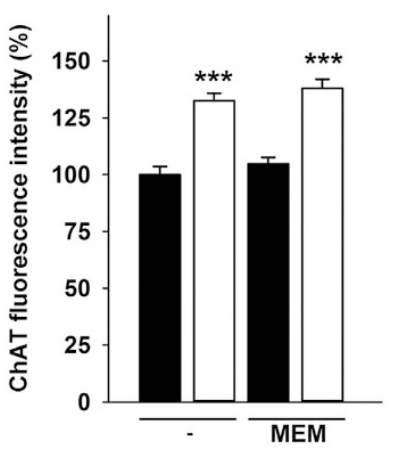

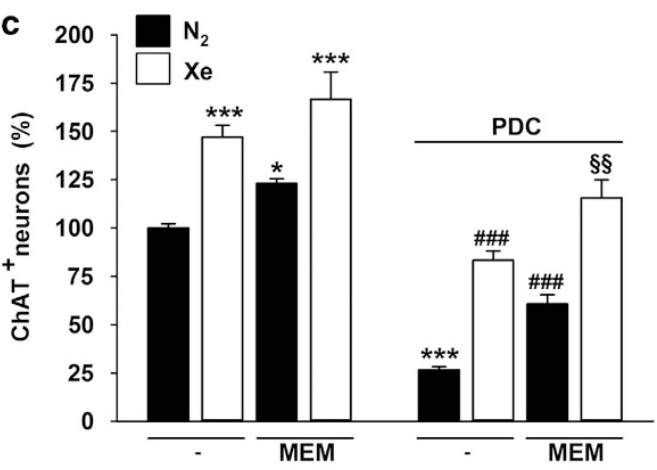

d
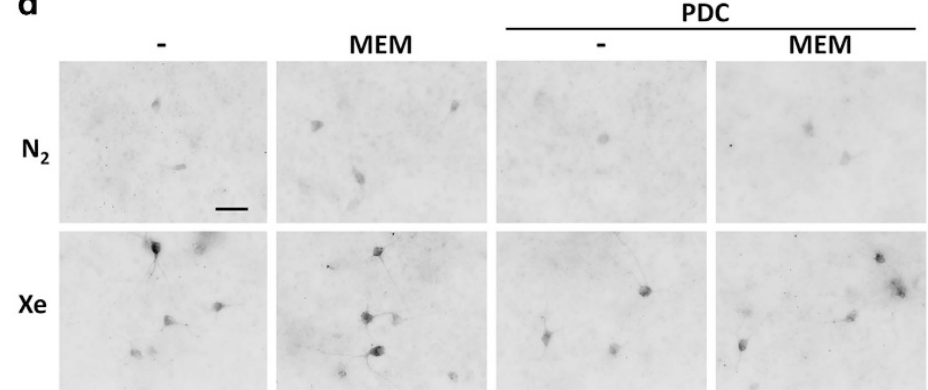

Figure 5. Xenon exerts both neurotrophic and neuroprotective effects for cholinergic neurons in septal cultures. (a) Left panel: Size of ChAT ${ }^{+}$ cell bodies in septal cultures exposed or not for 4 days to memantine (MEM; $10 \mu \mathrm{M}$ ) under a gas atmosphere containing $75 \% \mathrm{~N}_{2}$ or $75 \%$ Xe. Right panel: ChAT fluorescence intensity in $\mathrm{ChAT}^{+}$cell bodies exposed or not for 4 days to memantine (MEM; $\left.10 \mu \mathrm{M}\right)$ under a gas atmosphere containing $75 \% \mathrm{~N}_{2}$ or $75 \%$ Xe. Error bars indicate mean \pm S.E.M. ( $n=25$ randomly chosen neurons per condition). ${ }^{* * *} P<0.001$ relative to control cultures maintained under $75 \% \mathrm{~N}_{2}$ and ${ }^{\S} \mathrm{P}<0.05$ relative to cultures maintained under $75 \%$ Xe and to cultures maintained under $75 \%$ $\mathrm{N}_{2}$ in the presence of memantine. (b) Fluorescence digitized images presented under an inverted format to illustrate the impact of the same

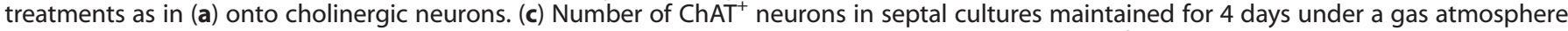
containing $75 \% \mathrm{~N}_{2}$ or $75 \%$ Xe and treated or not with PDC $(30 \mu \mathrm{M})$ in the presence or the absence of memantine $(10 \mu \mathrm{M})$. Error bars indicate mean \pm S.E.M. $(n=18)$. ${ }^{*} P<0.05,{ }^{* * * P}<0.001$ relative to control cultures maintained under $75 \% \mathrm{~N}_{2} ;{ }^{\# \# \#} P<0.001$ relative to PDC-treated cultures maintained under $75 \% \mathrm{~N}_{2}$ and ${ }^{\S \S} \mathrm{P}<0.01$ relative to PDC-treated cultures maintained in $75 \%$ Xe and PDC-treated cultures maintained in $75 \% \mathrm{~N}_{2}$ in the presence of memantine. (d) Fluorescence digitized images showing the impact of the same treatments as in (c) onto cholinergic neurons. Scale bars: $25 \mu \mathrm{m}$ and $50 \mu \mathrm{m}$ in (b) and (d), respectively.

Figure 5b). The intensity of ChAT immunolabelling was also significantly enhanced by the same gaseous treatment (Figure $5 \mathrm{a}$, right panel; Figure $5 \mathrm{~b})$. Memantine $(10 \mu \mathrm{M})$ produced a smaller increase of the size of $\mathrm{ChAT}^{+}$cell bodies than xenon and improved to some extent the effect that xenon exerted alone on this test parameter (Figure $5 \mathrm{a}$, left panel), indicating that a combination of the two treatments may better preserve the morphology of cholinergic neurons. Memantine did not increase on its own the intensity of ChAT immunostaining in cholinergic cell bodies and did not improve the effect that xenon exerted on this test parameter (Figure 5a, right panel).

Of interest, there was also a substantial increase (46\%) of neurons that were detectable by ChAT immunostaining in xenontreated cultures in comparison with cultures exposed to a nitrogen atmosphere (Figure $5 \mathrm{c})$. Memantine alone $(10 \mu \mathrm{M})$ increased $\mathrm{ChAT}^{+}$cell numbers in control cultures although to a lesser extent than xenon. The combination of the two treatments did not further increase cholinergic cell numbers in the absence of PDC (Figure 5c). When septal cultures were exposed to $30 \mu \mathrm{M}$ PDC between 12 and 16 DIV, only $26 \%$ of $\mathrm{ChAT}^{+}$neurons survived compared with control cultures maintained under a standard nitrogen atmosphere. Under an atmosphere enriched with xenon, the survival rate of $\mathrm{ChAT}^{+}$neurons was increased by more than threefold, reaching $>80 \%$ of control values. The increase in $\mathrm{ChAT}^{+}$ neuron survival was less important with memantine $(10 \mu \mathrm{M})$. Interestingly, when xenon and memantine treatments were combined under PDC exposure, the number of cholinergic neurons exceeded that found in control cultures.

\section{DISCUSSION}

We demonstrate for the first time that the noble gas xenon can provide partial but sustained protection to $A D$ vulnerable neurons (i.e., cortical neurons and septal cholinergic neurons) that undergo degeneration though chronic low-level excitotoxic stress. The protective effect of xenon was not reproduced with the other noble gas argon. We established that xenon interferes with a death pathway in which NMDA receptors and reactive oxygen species intervene crucially. Interestingly, xenon exerted cooperative neuroprotection with two noncompetitive NMDA receptor channel antagonists, memantine and ketamine. When evaluating the impact of xenon in septal cultures, we found that the noble gas was not only robustly neuroprotective for cholinergic neurons but that it also exerted potent trophic effects on them.

Xenon operates as a neuroprotectant against low-level excitotoxic stress

Previous studies have shown that xenon is neuroprotective in cellular or animal models that mimic acute brain ischemic or traumatic insults. ${ }^{7-11,43}$ The present work explored the neuroprotective potential of xenon in two model systems that mimic degenerative changes linked to the AD pathology.

In addition to histopathological hallmarks such as extracellular deposition of amyloid plaques and intracellular accumulation of neurofibrillary tangles, $A D$ is also characterized by a progressive loss of neurons in several brain areas, in particular the cerebral cortex. $^{28,30,44}$ We therefore established a model system of cortical 
cultures in which neuronal cell death was triggered by continued application of a synthetic glutamate analog PDC in order to generate slowly evolving excitotoxic stress, a likely component of neuronal death in the $A D$ pathology. ${ }^{24,45,46}$ As expected, excitotoxic cell death induced by PDC progressed relatively slowly in this experimental setting, presumably because neurodegenerative changes are caused by the progressive accumulation of endogenous glutamate in the extracellular milieu. ${ }^{33-35}$

When the conventional cell culture atmosphere was modified with a gas mixture, including the same amount of oxygen (20\%) and carbon dioxide $(5 \%)$ but $75 \%$ xenon instead of nitrogen, PDCinduced cortical cell death was reduced to a significant extent, 1 and 4 days after the onset of neurodegeneration. This observation is consistent with previous studies reporting on the neuroprotective effects of xenon in paradigms that mimic acute excitotoxic insults. ${ }^{7-11,43}$ However, to our knowledge, this is the first time that the neuroprotective potential of xenon is established in an experimental setting where neurodegenerative changes are progressively evolving. Incidentally, our data also show that control cortical neurons perfectly tolerate a long-term exposure to xenon which is coherent with brain histological studies carried out in newborn pigs inhaling the noble gas during extended periods of time. ${ }^{47}$

Xenon does not interfere with PDC effects on glutamate uptake or release

In agreement with previous reports, ${ }^{33-35}$ we found that PDC reduced the reuptake of glutamate and stimulated its release in the extracellular milieu. Hence, we hypothesized that neuroprotection by xenon against PDC could result from a reduced buildup of glutamate in the culture medium in response to the gaseous treatment. The effects of PDC on the uptake and release of glutamate were, however, not significantly impacted by xenon. The synaptic release of glutamate, which can be mimicked physiologically by a combined treatment with the $\mathrm{K}^{+}$channel blocker 4-aminopyridine and the $\mathrm{GABA}_{\mathrm{A}}$ receptor antagonist bicuculline, ${ }^{38,39}$ also remained unaffected under xenon exposure. This set of data clearly indicates that xenon is unlikely to afford protection by interfering with a presynaptic mechanism linked to the uptake or release of glutamate.

Xenon reduces NMDA receptor-mediated neurodegeneration

Memantine and ketamine, both of which operate as noncompetitive glutamate NMDA receptor antagonists, were able to mimic the protective effect of xenon against PDC, indicating that xenon was possibly acting itself by antagonism of this receptor subtype. Xenon is well known to reduce the activation of NMDA receptors, ${ }^{48}$ possibly by interfering at the binding site for glycine, ${ }^{14,21}$ a co-agonist for NMDA receptor activation, which modulates the amplitude and time course of glutamate-elicited responses. ${ }^{49,50}$ Xenon-mediated neuroprotection remained, however, unaffected when the culture medium used to maintain the cultures, that is, Neurobasal medium, was supplemented with an excess of glycine. This is rather unexpected because glycine has been reported previously to reverse neuroprotection by xenon in an in vitro model of traumatic brain injury. ${ }^{22}$ This result is, however, explainable considering the fact that glycine is present intrinsically in Neurobasal culture medium at a concentration of $400 \mu \mathrm{M}$, which is saturating for the NMDA receptor glycinebinding site. ${ }^{22}$ Even at saturating concentrations of glycine, xenon conserved the capacity to reduce NMDA currents, significantly. ${ }^{22}$ This suggests that NMDA receptors may still represent the neuroprotective target of xenon in the present paradigm but via a mechanism that is not sensitive to glycine and remains to be elucidated. Still consistent with the view that xenon operated via an antagonistic effect on NMDA receptors, the noble gas remained strongly protective when NMDA, the prototypical agonist of NMDA receptors was used instead of PDC to induce cortical cell death.

Unlike xenon, argon is not neuroprotective under chronic low-level excitotoxic conditions

Similar to xenon, argon has been reported to have strong organoprotective and neuroprotective effects. ${ }^{16,17}$ Neuroprotective effects have been described in a large variety of culture and animal models of acute excitotoxic stress. ${ }^{15}$ Therefore, the lack of protection of argon in the present study was rather unexpected. Unlike xenon, argon does not interfere with NMDA receptors. ${ }^{14,15}$ In fact, argon is believed to directly activate intracellular pathways that control the expression of anti-apoptotic proteins such as BCL-2. ${ }^{51}$ This may be one of the reason why argon is not neuroprotective in a context of chronic neurodegeneration.

Xenon provides cooperative rescue with memantine or ketamine Like xenon, the two noncompetitive NMDA antagonists, memantine and ketamine were protective in the present paradigm. This led us to evaluate the efficacy of treatments combining xenon with each of these two molecules. Most interestingly, we found that low concentrations of memantine and ketamine that afford no or partial protection on their own against PDC, had the capacity to markedly improve the rescue of cortical neurons provided by xenon, alone. This cooperative effect may be because of the fact that xenon on one hand, and memantine and ketamine on the other hand, operate via distinct mechanisms. Indeed, memantine and ketamine possess a blocking site situated within the NMDA channel pore, ${ }^{49,52}$ whereas xenon operates most likely elsewhere on the receptor. ${ }^{22}$ Cooperative effects of this kind may be of potential clinical interest, in particular in the case of memantine, which is an approved drug for the treatment of AD. ${ }^{27}$

Xenon prevents a death process involving oxidative stress

Oxidative stress participates actively in NMDA receptor-mediated neurodegeneration, ${ }^{53}$ which explains why pharmacological or genetic manipulations that restrain the production of reactive oxygen species have been reported to partially block neuronal death in acute models of excitototoxic stress. ${ }^{54,55}$ Here, we established that Trolox, a scavenger of hydroxyl radicals, ${ }^{40}$ and to a larger extent, NAC, a synthetic precursor of the intracellular antioxidant tripeptide glutathione, ${ }^{41}$ provided protection to cortical neurons in cultures chronically exposed to PDC. A number of gases including hydrogen, hydrogen sulfide and carbon monoxide, ${ }^{56-58}$ have been reported to possess antioxidant properties but experimental evidence is lacking in the case of xenon. The use of the cell-free DPPH assay revealed that xenon does not behave as an antioxidant in comparison with Trolox and NAC used as reference molecules. Therefore, one may assume that xenon prevented the propagation of oxidative stress-mediated damage, indirectly, through its capacity to limit NMDA receptor overstimulation.

Xenon exerts both neuroprotective and neurotrophic effects for septal cholinergic neurons

We were also interested in evaluating the impact of xenon onto basal forebrain septal cholinergic neurons, that is, a population of neurons that is also particularly vulnerable to degeneration in the context of the AD pathology. ${ }^{29,31,32}$ Xenon was not only robustly protective for cultured septal cholinergic neurons exposed to PDC but unexpectedly the gaseous treatment also had the capacity to strongly promote cholinergic neuron maturation in the absence of PDC. Most strikingly, xenon enhanced the size of cholinergic cell bodies and stimulated the cellular expression of the cholinergic marker protein ChAT in neurons immunopositive for this protein. The cholinotrophic effect of xenon extended presumably to a 
subset of dormant neurons expressing undetectable levels of the ChAT enzyme before initiation of the gaseous treatment. Indeed, the noble gas increased cholinergic cell numbers (i.e., $\mathrm{ChAT}^{+}$cells) by more than $40 \%$ in the absence of PDC. Even with a lower efficacy, memantine reproduced some of the cholinotrophic effects of xenon, indicating that NMDA receptor antagonism probably played a role in these effects. Present observations appear coherent with previous studies showing that NMDA receptor blockade can promote expression of cholinergic traits during development in subsets of forebrain glutamatergic neurons both in vitro and in vivo. .9,60 $^{-1}$

Quite remarkably, the trophic effects of xenon were observed despite the supplementation of the culture medium with nerve growth factor (NGF), a neuropeptide with cholinotrophic properties. ${ }^{61}$ This is not so surprising considering the fact that the NGF regimen used in this study $(3 \times 50 \mathrm{ng} / \mathrm{ml}$ over a period of 16 days) failed to promote full maturation of cholinergic neurons. This signifies that present culture conditions may be particularly favorable to reveal the cholinotrophic properties of xenon. The neurotrophic effects of xenon for cholinergic neurons are of interest as there is evidence from experimental lesions in animals and post-mortem human studies that these neurons are depleted of phenotypic markers well before dying in $A D .^{62,63}$ Whether induction or restoration of cholinergic traits can also occur with xenon in a pathological context remains, however, to be established.

As mentioned previously, xenon also provided robust protection to cholinergic neurons exposed to PDC. The noble gas was proportionally more efficacious to rescue cholinergic neurons than the bulk population of neurons that undergoes neurodegeneration in septal cultures (not shown) or in cortical cultures. This suggests that the trophic effects of xenon also occurred in PDCtreated septal cultures despite ongoing neurodegenerative changes. Coherent with this view, memantine, which was less effective than xenon in increasing the number of $\mathrm{ChAT}^{+}$neurons in control cultures, was also comparatively less efficacious than the noble gas to protect cholinergic neurons against PDC exposure.

Overall, our data demonstrate that the noble gas xenon exerts robust protective effects for cortical neurons and subcortical cholinergic neurons that are preferentially vulnerable in AD. Besides exerting true neuroprotective effects, xenon can also operate as a potent trophic factor for cholinergic neurons. Although any extrapolation from our in vitro work to a pathologic situation must be regarded as speculative, our data raise the intriguing possibility that xenon may have the potential to halt neurodegenerative events associated with the progression of the $A D$ pathology.

\section{MATERIALS AND METHODS}

\section{Pharmacological reagents}

The non-metabolizable analog of L-glutamate PDC, the preferential agonist of NMDA receptors NMDA and the NMDA receptor antagonist memantine, were all purchased from Tocris Biotechne (Lille, France). Ketamine was obtained from Virbac (Carros, France). The $\mathrm{GABA}_{\mathrm{A}}$ receptor antagonist bicuculline, the non-selective $\mathrm{K}^{+}$channel blocker 4-aminopyridine, NGF 2.5S, the two antioxidant molecules NAC, Trolox and the DPPH free radical were from Sigma-Aldrich (Saint-Quentin Fallavier, France).

\section{Cortical and septal neuronal cultures}

Animals were housed, handled and taken care of in accordance with recommendations of the Guide for the Care and Use of Laboratory Animals of the National Institutes of Health (NIH Publication no. 85-23, revised $1996)$ and the European Union Council Directives (86/609/CEE). Experimental procedures were authorized by the ethical committee on animal experiments Charles Darwin $n^{\circ} 5$.
Cortical cultures were prepared using gestational age 15.5 embryos harvested from Wistar female rats (Janvier LABS, Le Genest St Isle, France), which had been previously killed with a sodium pentobarbital overdose followed by cervical dislocation. Upon isolation, brain cortices were dissociated by mechanical dissociation in Leibovitz L15 culture medium (Sigma-Aldrich) according to the protocols previously described for mesencephalic cultures. ${ }^{64}$ Dissociated cells in suspension were then seeded at a density of $0.3-0.4 \times 10^{5}$ cells $/ \mathrm{cm}^{2}$ onto Nunc 48 -well multidish plates (Thermo Fisher Scientific, Roskilde, Denmark) pre-coated with $1 \mathrm{mg} / \mathrm{ml}$ polyethylenimine diluted in borate buffer, $\mathrm{pH}$ 8.3. Cultured cells were maintained for maturation in Neurobasal medium (Gibco, Saint Aubin, France) supplemented with a B27 cocktail minus antioxidants (Gibco), a N2 mix, $2 \mathrm{mM}$ glutamine and $100 \mathrm{IU} / \mathrm{ml}$ penicillin/ streptomycin. ${ }^{34}$ Glial cell proliferation was halted by adding $0.5 \mu \mathrm{M}$ of the antimitotic cytosine arabinoside, not later than $12 \mathrm{~h}$ after plating.

Septal cultures were obtained by dissection of the forebrain area that contains cholinergic neurons and gives rise to the medial septal nucleus and the nucleus of the diagonal band of Brocca. ${ }^{61,66}$ After trituration of brain tissue pieces, septal cells in suspension were plated at a density of $1-1.2 \times 10^{5}$ cells $/ \mathrm{cm}^{2}$. Septal cultures were maintained using the same conditions as those described for cortical cultures, except that NGF $(50 \mathrm{ng} / \mathrm{ml})$ was added to the cultures after plating, and then at DIV 7 and 12 to stimulate the phenotypic differentiation of cholinergic neurons. ${ }^{61,65}$ Cortical and septal cultures were maintained up to the indicated times before being processed for assessment of test parameters.

\section{Culture exposure to gases}

After pharmacological treatments, 48-well multi-dish plates containing cultured cells were disposed above a metallic base plate receiving atop of it a Plexiglas incubation chamber. The two pieces were then screwed together to produce an air-tight seal. Humidification of the chamber was achieved by disposing a multi-dish plate wherein culture wells filled with distilled water were placed directly in contact with the inside atmosphere. When needed, the pre-defined gas mixture comprising $20 \% \mathrm{O}_{2}, 5 \% \mathrm{CO}_{2}$ and $75 \%$ of the test gas was injected at a flow rate of $\sim 10 \mathrm{l} / \mathrm{min}$ into the incubation chamber with the inlet and outlet valves open. When the $\mathrm{CO}_{2}$ concentration measured at the outlet with a gas analyzer reached $5 \%$, the injection was stopped and the chamber was sealed by turning off the inlet and outlet valves. The incubation chamber was then placed in a conventional incubator at $37{ }^{\circ} \mathrm{C}$ for incubation times indicated in the manuscript. We generally used pre-defined gas mixtures provided by Air Liquide (France). In some experiments, controlled gas atmospheres were also delivered to incubation chambers using a gas mixing system (GasMix, Alytech, Juvisy/Orge, France) supplied by tanks filled by individual pure gases (Air Liquide).

\section{Protein detection by immunofluorescence}

The cultures, fixed with $4 \%$ formaldehyde in Dulbecco's phosphatebuffered saline (PBS), were washed twice with PBS before an incubation step at $4{ }^{\circ} \mathrm{C}$ for $48-96 \mathrm{~h}$ with primary antibodies diluted in PBS containing $0.2 \%$ Triton X-100 to improve cell membrane permeability. A monoclonal MAP-2 antibody (clone AP-20, \#M2320; Sigma-Aldrich) diluted 1/200 was used as the pan-neuronal marker and a goat anti-ChAT affinity purified antibody diluted 1/100 (\#AB144P; Merck Millipore, Darmstadt, Germany) as the cholinergic marker. Detection of primary antibodies was performed with an Alexa Fluor-555 conjugate of an anti-mouse lgG antibody (1/500) or an Alexa Fluor-555 conjugate of an anti-goat antibody (1/1000) both obtained from Life Technologies (Saint Aubin, France).

\section{Assessment of neuronal viability}

The survival of all neurons regardless of their neurotransmitter phenotype was monitored by counting MAP-2 ${ }^{+}$cells. Briefly, fluorescent images of cultured cells were acquired with an Eclipse TE-2000 inverted fluorescent microscope (Nikon, Champigny-sur-Marne, France) equipped with an ORCA-ER digital camera (Hamamatsu Photonics, Massy, France) operated with the $\mathrm{HCl}$ software (Hamamatsu Corp., Bridgewater, NJ, USA). In each culture well, 10 digitized images randomly acquired with a $\times 10$ objective were taken for cell counting using the image processing program Image J. Counting of $\mathrm{ChAT}^{+}$neurons was performed on digitized images acquired with a $\times 10$ objective mounted onto an Arrayscan XTi automated workstation equipped with the HCS Studio Software (Thermofisher Scientific, Courtaboeuf, France). Mosaic reconstruction of partially 
overlapping digitized images allowed the counting of $\mathrm{ChAT}^{+}$neurons over $>60 \%$ of the surface area of each culture well.

\section{Markers of cholinergic neuron maturation}

To estimate the impact that test treatments could have on the maturation of cholinergic neurons, digitized images of $\mathrm{ChAT}^{+}$neurons were taken randomly with the Nikon Eclipse TE-2000 microscope using a $\times 40$ objective and identical acquisition parameters. Images were used to quantify the surface area and the fluorescence intensity of positive cell bodies using the MCID analysis software (InterFocus Imaging Ltd, Cambridge, UK). Specific ChAT fluorescence intensity was corrected by subtracting background intensity levels for each treatment condition. All results were expressed in percent of control values.

\section{$\left[{ }^{3} \mathrm{H}\right]-\mathrm{D}$-aspartate uptake and efflux assays}

$\left[{ }^{3} \mathrm{H}\right]$-D-aspartate, a non-metabolizable analog of L-glutamate that labels the cytosolic and vesicular pools of endogenous excitatory amino acids, ${ }^{3,66}$ was used to estimate the uptake and release of glutamate. Briefly, the culture medium was removed and the cultures were allowed to recover for $5 \mathrm{~min}$ at $37^{\circ} \mathrm{C}$ in PBS supplemented with $5 \mathrm{mM}$ glucose before further processing. The uptake was initiated by the addition of $1 \mu \mathrm{l}$ of $\left[2,3-{ }^{3} \mathrm{H}\right]$-D-aspartate $(13 \mathrm{Ci} / \mathrm{mmol}$; Perkin Elmer, Waltham, MA, USA) to culture wells exposed or not to different test treatments in the presence of gas atmospheres enriched or not with xenon. After $30 \mathrm{~min}$, the cultures were washed twice with cold PBS to remove the tritiated amino acid in excess and the radioactivity accumulated intracellularly was recovered by scrapping off cells in distilled water. Samples were then assessed by liquid scintillation spectrometry. The release of $\left[2,3-{ }^{3} \mathrm{H}\right]-\mathrm{D}$-aspartate was carried out in cultures that were pre-loaded for $30 \mathrm{~min}$ with the tritiated amino acid, washed twice with PBS and then exposed to various test treatments in the presence of gas atmospheres enriched or not with xenon. The fraction of radioactivity recovered in the incubation medium during the next $30 \mathrm{~min}$ was collected for assessment by liquid scintillation spectrometry.

\section{Free radical-scavenging assay}

Radical-scavenging activities were measured using DPPH, a hydrazyl derivative producing a stable free radical with a deep purple color when diluted in pure ethanol. ${ }^{41}$ Briefly, $225 \mu \mathrm{l}$ of a DPPH ethanol solution $(0.025 \mathrm{mg} / \mathrm{ml})$ were distributed into individual wells of a Nunc $96-$ well microplate. The volume was then completed to $250 \mu \mathrm{l}$ by addition of the test compounds diluted in ethanol or by ethanol, alone. Blanks contained $250 \mu \mathrm{l}$ of pure ethanol instead of DPPH. The microplate shaken vigorously during $10 \mathrm{~s}$ was then allowed to stand for $1 \mathrm{~h}$ in the dark at room temperature under atmospheres containing either $75 \%$ nitrogen or $75 \%$ xenon. This was followed by measurement of the absorbance at $517 \mathrm{~nm}$ using a Spectramax M4 multiplate reader (Molecular Devices, Sunnyvale, CA, USA). Blank values obtained by replacing DPPH with pure ethanol were subtracted from test samples.

\section{Statistical analyses}

Data expressed as means \pm S.E.M were analyzed using the SigmaPlot 12.5 software (Systat Software Inc, San Jose, CA, USA). Multiple comparisons against a single reference group were made by one-way ANOVA with Dunnett's test post hoc analysis. When all pairwise comparisons were required, one-way ANOVA was followed by the Student-Newman-Keuls post hoc test.

\section{ABBREVIATIONS}

$A D$, Alzheimer's disease; ChAT, Choline acetyltransferase; DIV, day in vitro; DPPH, 2-diphenyl-1-picrylhydrazyl; MAP-2, microtubule-associated protein; NAC, N-acetylcysteine; NGF, Nerve growth factor; NMDA, N-methyl-Daspartate; PBS, Dulbecco's phosphate-buffered saline; PDC, L-transpyrrolidine-2,4-dicarboxylic acid

\section{ACKNOWLEDGEMENTS}

The research leading to these results was supported by program Investissements d'Avenir [ANR-10-IAIHU-06] and Translational Research Infrastructure for Biotherapies in Neurosciences [ANR-11-INBS-0011-NeurATRIS]. This study was funded by a grant from Air Liquide Santé International. We thank Géraldine Farjot for helpful comments on the manuscript. We are grateful to Matthieu Chalopin for coordinating operational aspects of the project and to François Gilles for his expertise with gas delivery systems. We also wish to acknowledge the valuable assistance of David Akbar from the CELIS cell culture core facility, regarding fluorescence microscopy analysis.

\section{COMPETING INTERESTS}

ML and JP are Air Liquide employees. DLN is recipient of a CIFRE fellowship (2014/ 0589) co-funded by Association Nationale de la Recherche et de la Technologie (ANRT) and Air Liquide Santé International.

\section{REFERENCES}

1 Jordan BD, Wright EL. Xenon as an anesthetic agent. AANA J 2010; 78: 387-392.

2 Stoppe C, Rimek A, Rossaint R, Rex S, Stevanovic A, Schälte G et al. Xenon consumption during general surgery: a retrospective observational study. Med Gas Res 2013; 3: 12.

3 Ma D, Lim T, Xu J, Tang H, Wan Y, Zhao H et al. Xenon preconditioning protects against renal ischemic-reperfusion injury via HIF-1a activation. J Am Soc Nephrol 2009; 20: 713-720.

4 Li Q, Lian C, Zhou R, Li T, Xiang X, Liu B. Pretreatment with xenon protected immature rabbit heart from ischaemia/reperfusion injury by opening of the mitoKATP channel. Heart Lung Circ 2013; 22: 276-283.

5 Hobbs C, Thoresen M, Tucker A, Aquilina K, Chakkarapani E, Dingley J. Xenon and hypothermia combine additively, offering long-term functional and histopathologic neuroprotection after neonatal hypoxia/ischemia. Stroke 2008; 39: 1307-1313.

6 Faulkner S, Bainbridge A, Kato T, Chandrasekaran M, Kapetanakis AB, Hristova M et al. Xenon augmented hypothermia reduces early lactate/ $\mathrm{N}$-acetylaspartate and cell death in perinatal asphyxia. Ann Neurol 2011; 70: 133-150.

7 Banks P, Franks NP, Dickinson R. Competitive inhibition at the glycine site of the $\mathrm{N}$-methyl-D-aspartate receptor mediates xenon neuroprotection against hypoxia-ischemia. Anesthesiology 2010; 112: 614-622.

8 David HN, Leveille F, Chazalviel L, MacKenzie ET, Buisson A, Lemaire $M$ et al. Reduction of ischemic brain damage by nitrous oxide and xenon. J Cereb Blood Flow Metab 2003; 23: 1168-1173.

9 Luo Y, Ma D, leong E, Sanders RD, Yu B, Hossain M, Maze M. Xenon and sevoflurane protect against brain injury in a neonatal asphyxia model. Anesthesiology 2008; 109: 782-789.

10 Thoresen M, Hobbs CE, Wood T, Chakkarapani E, Dingley J. Cooling combined with immediate or delayed xenon inhalation provides equivalent long-term neuroprotection after neonatal hypoxia-ischemia. J Cereb Blood Flow Metab 2009; 29: 707-714.

11 Natale G, Cattano D, Abramo A, Forfori F, Fulceri F, Fornai F et al. Morphological evidence that xenon neuroprotects against $\mathrm{N}$-methyl-DL-aspartic acid-induced damage in the rat arcuate nucleus: a time-dependent study. Ann $N$ Y Acad Sci 2006; 1074: 650-658.

12 Yamamoto $Y$, Kawaguchi M, Kurita N, Kakimoto M, Inoue S, Furuya H. Effects of xenon on ischemic spinal cord injury in rabbits: a comparison with propofol. Acta Anaesthesiol Scand 2010; 54: 337-342.

13 Campos-Pires R, Armstrong SP, Sebastiani A, Luh C, Gruss M, Radyushkin K et al. Xenon improves neurologic outcome and reduces secondary injury following trauma in an in vivo model of traumatic brain injury. Crit Care Med 2015; 43: 149-158.

14 Harris K, Armstrong SP, Campos-Pires R, Kiru L, Franks NP, Dickinson R. Neuroprotection against traumatic brain injury by xenon, but not argon, is mediated by inhibition at the $\mathrm{N}$-methyl-D-aspartate receptor glycine site. Anesthesiology 2013; 119: 1137-1148.

15 Höllig A, Schug A, Fahlenkamp AV, Rossaint R, Coburn M. Argon Organo-Protective Network (AON). Argon: systematic review on neuro- and organoprotective properties of an "inert" gas. Int J Mol Sci 2014; 15: 18175-18196.

16 David HN, Haelewyn B, Degoulet M, Colomb Jr DG, Risso JJ, Abraini JH. Ex vivo and in vivo neuroprotection induced by argon when given after an excitotoxic or ischemic insult. PLoS One 2003; 7: e30934.

17 Ryang YM, Fahlenkamp AV, Rossaint R, Wesp D, Loetscher PD, Beyer C et al. Neuroprotective effects of argon in an in vivo model of transient middle cerebral artery occlusion in rats. Crit Care Med 2011; 39: 1448-1453.

18 Ulbrich F, Schallner N, Coburn M, Loop T, Lagrèze WA, Biermann J et al. Argon inhalation attenuates retinal apoptosis after ischemia/reperfusion injury in a time- and dose-dependent manner in rats. PLoS One 2014; 9: e115984.

19 Bantel C, Maze M, Trapp S. Neuronal preconditioning by inhalational anesthetics: evidence for the role of plasmalemmal adenosine triphosphate-sensitive potassium channels. Anesthesiology 2009; 110: 986-995. 
20 Gruss M, Bushell TJ, Bright DP, Lieb WR, Mathie A, Franks NP. Two-pore-domain $\mathrm{K}^{+}$channels are a novel target for the anesthetic gases xenon, nitrous oxide, and cyclopropane. Mol Pharmacol 2004; 65: 443-452.

21 Armstrong SP, Banks PJ, McKitrick TJ, Geldart CH, Edge CJ, Babla R et al. Identification of two mutations (F758W and F758Y) in the N-methyl-D-aspartate receptor glycine-binding site that selectively prevent competitive inhibition by xenon without affecting glycine binding. Anesthesiology 2012; 117: 38-47.

22 Dickinson R, Peterson BK, Banks P, Simillis C, Martin JC, Valenzuela CA et al. Competitive inhibition at the glycine site of the $\mathrm{N}$-methyl-D-aspartate receptor by the anesthetics xenon and isoflurane: evidence from molecular modeling and electrophysiology. Anesthesiology 2007; 107: 756-767.

23 Spaggiari S, Kepp O, Rello-Varona S, Chaba K, Adjemian S, Pype J et al. Antiapoptotic activity of argon and xenon. Cell Cycle 2013; 12: 2636-2642.

24 Mattson MP, Chan SL. Neuronal and glial calcium signaling in Alzheimer's disease. Cell Calcium 2003; 34: 385-397.

25 Majláth Z, Toldi J, Vécsei L. The potential role of kynurenines in Alzheimer's disease: pathomechanism and therapeutic possibilities by influencing the glutamate receptors. J Neural Transm 2014; 121: 881-889.

26 Talantova M, Sanz-Blasco S, Zhang X, Xia P, Akhtar MW, Okamoto S et al. A induces astrocytic glutamate release, extrasynaptic NMDA receptor activation, and synaptic loss. Proc Natl Acad Sci USA 2013; 110: E2518-E2527.

27 McShane R, Areosa Sastre A, Minakaran N. Memantine for dementia. Cochrane Database Syst Rev 2006; 2: CD003154.

28 Gomez-Isla T, Price JL, McKeel Jr DW, Morris JC, Growdon JH, Hyman BT. Profound loss of layer II entorhinalcortex neurons occurs in very mild Alzheimer's disease. J Neurosci 1996; 16: 4491-4500.

29 Mesulam M. The cholinergic lesion of Alzheimer's disease: pivotal factor or side show? Learn Mem 2004; 11: 43-49.

30 Andrade-Moraes CH, Oliveira-Pinto AV, Castro-Fonseca E, da Silva CG, Guimarães DM, Szczupak D et al. Cell number changes in Alzheimer's disease relate to dementia, not to plaques and tangles. Brain 2013; 136: 3738-3752.

31 Perry EK, Tomlinson BE, Blessed G, Perry RH, Cross AJ, Crow TT. Noradrenergic and cholinergic systems in senile dementia of Alzheimer type. Lancet 1981; 2: 149 .

32 Vogels OJ, Broere CA, ter Laak HJ, ten Donkelaar HJ, Nieuwenhuys R, Schulte BP. Cell loss and shrinkage in the nucleus basalis Meynert complex in Alzheimer's disease. Neurobiol Aging 1990; 11: 3-13.

33 Waagepetersen HS, Shimamoto K, Schousboe A. Comparison of effects of DL-threo-beta-benzyloxyaspartate (DL-TBOA) and L-trans-pyrrolidine-2,4-dicarboxylate (t-2,4-PDC) on uptake and release of [3H]-D-aspartate in astrocytes and glutamatergic neurons. Neurochem Res 2001; 26: 661-666.

34 Nafia I, Re DB, Masmejean F, Melon C, Kachidian P, Kerkerian-Le Goff L et al. Preferential vulnerability of mesencephalic dopamine neurons to glutamate transporter dysfunction. J Neurochem 2008; 105: 484-496.

35 Gouix E, Léveillé $F$, Nicole $O$, Melon C, Had-Aissouni L, Buisson A. Reverse glial glutamate uptake triggers neuronal cell death through extrasynaptic NMDA receptor activation. Mol Cell Neurosci 2009; 40: 463-473.

36 Liu LT, Xu Y, Tang P. Mechanistic insights into xenon inhibition of NMDA receptors from MD simulations. J Phys Chem B 2010; 114: 9010-9016.

37 Jensen JB, Pickering DS, Schousboe A. Depolarization-induced release of $\left[{ }^{3} \mathrm{H}\right]$-D-aspartate from GABAergic neurons caused by reversal of glutamate transporters. Int J Dev Neurosci 2000; 18: 309-315.

38 Hardingham GE, Fukunaga Y, Bading H. Extrasynaptic NMDARs oppose synaptic NMDARs by triggering CREB shut-off and cell death pathways. Nat Neurosci 2002; 5: 405-414.

39 Hoey SE, Williams RJ, Perkinton MS. Synaptic NMDA receptor activation stimulates alpha-secretase amyloid precursor protein processing and inhibits amyloid-beta production. J Neurosci 2009; 29: 4442-4460.

40 Guerreiro S, Ponceau A, Toulorge D, Martin E, Alvarez-Fischer D, Hirsch EC et al. Protection of midbrain dopaminergic neurons by the end-product of purine metabolism uric acid: potentiation by low-level depolarization. J Neurochem 2009; 109: 1118-1128.

41 Samuni Y, Goldstein S, Dean OM, Berk M. The chemistry and biological activities of N-acetylcysteine. Biochim Biophys Acta 2013; 1830: 4117-4129.

42 Matsunaga N, Tsuruma K, Shimazawa M, Yokota S, Hara H. Inhibitory actions of bilberry anthocyanidins on angiogenesis. Phytother Res 2010; 24: S42-S47.

43 Deng J, Lei $C$, Chen $Y$, Fang $Z$, Yang Q, Zhang $H$ et al. Neuroprotective gases-fantasy or reality for clinical use? Prog Neurobiol 2014; 115: 210-245.

44 Serrano-Pozo A, Frosch MP, Masliah E, Hyman BT. Neuropathological alterations in Alzheimer disease. Cold Spring Harb Perspect Med 2011; 1: a006189.
45 Ong WY, Tanaka K, Dawe GS, Ittner LM, Farooqui AA. Slow excitotoxicity in Alzheimer's disease. J Alzheimers Dis 2013; 35: 643-668.

46 Parsons MP, Raymond LA. Extrasynaptic NMDA receptor involvement in central nervous system disorders. Neuron 2014; 82: 279-293.

47 Sabir H, Bishop S, Cohen N, Maes E, Liu X, Dingley J et al. Neither xenon nor fentanyl induces neuroapoptosis in the newborn pig brain. Anesthesiology 2013; 119: $345-357$.

48 Franks NP, Dickinson R, de Sousa SL, Hall AC, Lieb WR. How does xenon produce anaesthesia? Nature 1998; 396: 324.

49 Lipton SA. Pathologically-activated therapeutics for neuroprotection: mechanism of NMDA receptor block by memantine and S-nitrosylation. Curr Drug Targets 2007; 8: 621-632.

50 Cummings KA, Popescu GK. Glycine-dependent activation of NMDA receptors. $J$ Gen Physiol 2015; 145: 513-527.

51 Zhuang L, Yang T, Zhao H, Fidalgo AR, Vizcaychipi MP, Sanders RD et al. The protective profile of argon, helium, and xenon in a model of neonatal asphyxia in rats. Crit Care Med 2012; 40: 1724-1730.

52 Kotermanski SE, Johnson JW. $\mathrm{Mg}^{2+}$ imparts NMDA receptor subtype selectivity to the Alzheimer's drug memantine. J Neurosci 2009; 29: 2774-2779.

53 Coyle JT, Puttfarcken P. Oxidative stress, glutamate, and neurodegenerative disorders. Science 1993; 262: 689-695.

54 Brennan AM, Suh SW, Won SJ, Narasimhan P, Kauppinen TM, Lee H et al. NADPH oxidase is the primary source of superoxide induced by NMDA receptor activation. Nat Neurosci 2009; 12: 857-863.

55 Girouard H, Wang G, Gallo EF, Anrather J, Zhou P, Pickel VM et al. NMDA receptor activation increases free radical production through nitric oxide and NOX2. J Neurosci 2009; 29: 2545-2552.

56 Mohanakumar KP, Thomas B, Sharma SM, Muralikrishnan D, Chowdhury R Chiueh CC. Nitric oxide: an antioxidant and neuroprotector. Ann N Y Acad SCi 2002; 962: 389-401.

57 Ohsawa I, Ishikawa M, Takahashi K, Watanabe M, Nishimaki K, Yamagata K et al. Hydrogen acts as a therapeutic antioxidant by selectively reducing cytotoxic oxygen radicals. Nat Med 2007; 13: 688-694.

58 Kimura Y, Goto Y, Kimura H. Hydrogen sulfide increases glutathione production and suppresses oxidative stress in mitochondria. Antioxid Redox Signal 2010; 12: $1-13$.

59 Liu X, Popescu IR, Denisova JV, Neve RL, Corriveau RA, Belousov AB. Regulation of cholinergic phenotype in developing neurons. I Neurophysiol 2008; 99: 2443-2455.

60 Guemez-Gamboa A, Xu L, Meng D, Spitzer NC. Non-cell-autonomous mechanism of activity-dependent neurotransmitter switching. Neuron 2014; 82: 1004-1016.

61 Hartikka J, Hefti F. Development of septal cholinergic neurons in culture: plating density and glial cells modulate effects of NGF on survival, fiber growth, and expression of transmitter-specific enzymes. J Neurosci 1988; 8: 2967-2985.

62 Strada O, Vyas S, Hirsch EC, Ruberg M, Brice A, Agid Y et al. Decreased choline acetyltransferase mRNA expression in the nucleus basalis of Meynert in Alzheimer disease: an in situ hybridization study. Proc Natl Acad Sci USA 1992; 89: 9549-9553.

63 Mufson EJ, Counts SE, Perez SE, Ginsberg SD. Cholinergic system during the progression of Alzheimer's disease: therapeutic implications. Expert Rev Neurother 2008; 8: 1703-1718.

64 Toulorge D, Guerreiro S, Hild A, Maskos U, Hirsch EC, Michel PP. Neuroprotection of midbrain dopamine neurons by nicotine is gated by cytoplasmic $\mathrm{Ca}^{2+}$. FASEB $\mathrm{J}$ 2011; 25: 2563-2573.

65 Traver S, Salthun-Lassalle B, Marien M, Hirsch EC, Colpaert F, Michel PP. The neurotransmitter noradrenaline rescues septal cholinergic neurons in culture from degeneration caused by low-level oxidative stress. Mol Pharmacol 2005; 67: 1882-1891.

66 Douhou A, Troadec JD, Ruberg M, Raisman-Vozari R, Michel PP. Survival promotion of mesencephalic dopaminergic neurons by depolarizing concentrations of $\mathrm{K}^{+}$requires concurrent inactivation of NMDA or AMPA/kainate receptors. Neurochem 2001; 78: 163-174.

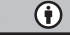

This work is licensed under a Creative Commons Attribution 4.0 International License. The images or other third party material in this article are included in the article's Creative Commons license, unless indicated otherwise in the credit line; if the material is not included under the Creative Commons license, users will need to obtain permission from the license holder to reproduce the material. To view a copy of this license, visit http://creativecommons.org/licenses/ by/4.0/ 